\title{
Avaliaçáo da capacidade funcional de adolescentes portadores de Mucopolissacaridose do tipo II
}

\author{
Ingrid Alves Barros Silva Amaral ${ }^{a}$, Reinaldo Luna de Omena Filhob, \\ Joáo Ancelmo dos Reis Neto ${ }^{c}$, Monique Carla da Silva Reis ${ }^{b}$

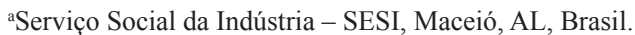 \\ bUniversidade Estadual de Ciências da Saúde de Alagoas - UNCISAL, Maceió, AL, Brasil. \\ ${ }^{\mathrm{c}}$ Centro Universitário Tiradentes - UNIT, Maceió, AL, Brasil.
}

\begin{abstract}
Resumo: Introdução: A Mucopolissacaridose II (MPS II) é uma doença genética, ligada ao cromossomo X e que afeta quase que exclusivamente o sexo masculino. Apresenta-se de forma multissistêmica, ocasionada pelo acúmulo progressivo de glicosaminoglicanos (GAG) em tecidos e órgãos, o que causa atraso no desenvolvimento neuropsicomotor, ocasionando limitações na realização de atividades de vida diária. Objetivo: O objetivo deste estudo foi a identificação das áreas de desempenho nas quais os portadores de MPS II apresentam maior comprometimento, além da análise do perfil socioeconômico da respectiva família. Método: A pesquisa se deu através de entrevista com a responsável por três irmãos adolescentes, A1-19 anos, A2-16 anos e A3-15anos, portadores de MPS II, no Estado de Alagoas. A coleta foi efetuada por profissional capacitado, utilizando a Medida de Independência Funcional (MIF) como questionário para identificação de perdas funcionais, e ABEP, para análise do perfil socioeconômico. Resultados: Os resultados apontaram que, em termos socioeconômicos, a família está inserida na classe $\mathrm{C} 1$. Em relação ao desempenho, verificou-se que, entre os irmãos, A1 apresenta dependência total nas habilidades investigadas; A2 apresenta perdas funcionais mais incapacitantes, necessitando de auxílio moderado, e A3 possui boa parte das habilidades físicas e cognitivas preservada, porém possui dependência modificada. Conclusão: Diante das dificuldades identificadas neste estudo, foi analisada a importância do diagnóstico precoce e das terapias de reabilitação aliadas à terapia enzimática, para que haja um retardo na progressão da doença.
\end{abstract}

Palavras-chave: Mucopolissacaridose II, Reabilitação, Terapia Ocupacional.

\section{Assessment of functional capacity of teens who are Mucopolysaccharidosis type II carriers}

\begin{abstract}
Introduction: Mucopolysaccharidosis II (MPS II) is an X-linked genetic disease which almost exclusively affects males. The disease presents a multisystem form, caused by the progressive accumulation of glycosaminoglycans (GAGs) in tissues and organs. It causes developmental delays resulting in limitations on the performance of day-to-day activities. Objective: The aim of this study was to identify occupational performance areas in which people with MPS II have greater impairment and analyze their family's socioeconomic profile. Method: The research was done through interviews with an individual responsible for three adolescent brothers, (A1-19 years, A2-16 years and A3-15 years) who are MPS II carriers from Alagoas, Brazil. Data collection was performed by a trained professional using the Functional Independence Measure (FIM) as a questionnaire to identify the functional losses and the ABEP for socioeconomic profile analysis. Results: The results showed that the family is included in the $\mathrm{C} 1$ socioeconomic class. Among the siblings, A1 presents total dependence on the investigated skills; A2 presents the most disabling functional losses requiring moderate assistance and A3 has much of his physical and cognitive skills preserved, but has modified dependence. Conclusion: Taking into consideration the difficulties identified in this study, we highlighted the importance of early diagnosis and rehabilitation therapies combined with enzyme therapy, which can delay disease progression.
\end{abstract}

Keywords: Mucopolysaccharidosis II, Reabilitation, Occupational Therapy.

Autor para correspondência: Monique Carla da Silva Reis, Universidade Estadual de Ciências da Saúde de Alagoas, Rua Doutor Jorge de Lima, 113, Trapiche da Barra, CEP 57010-300, Maceió, AL, Brasil, e-mail: moniquecsto@gmail.com

Recebido em Maio 19, 2016; $1^{\text {a }}$ Revisão em Jul. 6, 2016; 2ª Revisão em Ago. 4, 2016; Aceito em Set. 9, 2016. 


\section{Introdução}

Segundo Camelier (2001), os erros inatos do metabolismo (EIM) são doenças determinadas geneticamente, causadas por um defeito enzimático específico, que leva ao bloqueio de uma determinada rota metabólica.

Atualmente, a classificação mais utilizada divide as mucopolissacaridoses em sete subtipos, com base nos achados clínicos e testes laboratoriais (NEUFELD; MUENZER, 2001). Mesmo apresentando características crônicas e progressivas típicas, as manifestaçóes clínicas de cada subtipo variam de acordo com a enzima que está ausente no portador da doença, o que determina o tipo de mucopolissacaridose (ROCHA et al., 2012).

Charles Hunter fez o primeiro relato sobre mucopolissacaridose do tipo II (MPS II) em 1917, descrevendo características específicas desse tipo de MPS (JONES, 1998).

As Mucopolissacaridoses (MPS) são um grupo de doenças genéticas causadas pela deficiência de uma das enzimas lisossômicas responsáveis por uma etapa específica na degradação dos glicosaminoglicanos (GAG). Esta apresenta herança ligada ao $\mathrm{X}$, sendo causada pela atividade deficiente da enzima iduronato sulfatase, com consequente aumento da concentração urinária dos GAG dermatan sulfato e heparan sulfato (PINTO et al., 2006, p. 274).

A MPS II é o tipo mais frequente no Brasil e a maior parte das pessoas acometidas é do sexo masculino, havendo também relatos de ocorrência no sexo feminino. É rara e caracterizada ou por uma translocação anormal ou uma inativação não aleatória do cromossomo X (TUSCH et al., 2004 apud PEREIRA et al., 2011). Boy e Schwart (2011) afirmam que a MPS II tem incidência aproximada de 0,31 a 0,71 por 100 mil nascidos vivos.

Vale ressaltar o caráter genético e progressivo da MPS II, que provoca risco de recorrência numa mesma família e até na mesma prole, o que torna imprescindível a orientação e o acompanhamento genético para famílias que já têm um membro diagnosticado (ROCHA et al., 2012).

Segundo Boy e Schwart (2011), a mucopolissacaridose II se caracteriza por manifestaçóes precoces, que consistem em atraso no desenvolvimento da linguagem, distúrbio de comportamento, performance escolar regular e retardo mental. As alteraçóes esqueléticas consistem de más formaçôes esqueléticas e vertebrais, e rigidez articular. Comprometimento respiratório e hepatoesplenomegalia, sendo que hérnias umbilicais e inguinais também são frequentes. Alteraçóes em pele e cardiológicas se manifestam com frequência.

É importante realizar o diagnóstico precoce da Mucopolissacaridose por análise enzimática e estudos moleculares, a fim de permitir um adequado acompanhamento genético e um apoio para a família em estágio inicial da doença (PEREIRA et al., 2011).

O diagnóstico diferencial deve ser feito com as demais mucopolissacaridoses, além de outras doenças lisossômicas; para isso, é necessário realizar exames complementares e dosagens enzimáticas específicas (PINTO, 2005).

Não existe tratamento curativo para as MPS. As opçôes terapêuticas disponíveis incluem intervençóes realizadas no nível do fenótipo clínico, tais como cirurgias para correção de hérnias e adenoides, suporte ventilatório noturno, etc (BOY; SCHWART, 2011). O tratamento da MPS II é sintomático e de suporte, mas há relatos da utilização de corticoides e de transplante de medula óssea; porém, hoje em dia, o mais comum é a reposição enzimática, pela qual a criança recebe as enzimas que são deficitárias em seu organismo (PEREIRA et al., 2011).

De acordo com Giugliani et al. (2010), o acúmulo de GAG ocorre em vários órgãos e tecidos nos portadores de Mucopolissacaridose II, o que resulta em uma série de sinais e sintomas, integrantes de um quadro clínico multissistêmico, que compromete ossos e articulações, vias respiratórias, sistema cardiovascular e muitos outros órgãos e tecidos, incluindo, em alguns casos, comprometimento das funçóes cognitivas. De acordo com Guarany (2011), estas manifestaçôes clínicas causam diversas limitaçôes nas atividades de vida diária (AVD) destes pacientes. O desenvolvimento de doenças crônicas durante a vida pode resultar em uma perda contínua de funçóes orgânicas e biológicas, que podem levar à incapacidade funcional.

Para Neri (2001), a independência funcional é a capacidade de realizar algo com os próprios meios, ou seja, sem interferência de outras pessoas ou de meios de auxílio. Está ligada à mobilidade e à capacidade funcional, para as quais o indivíduo não requer ajuda para a realização das atividades de vida diária, ou seja, a independência supóe condiçóes motoras e cognitivas satisfatórias para o desempenho destas tarefas.

Complementando este pensamento, a Organização Mundial da Saúde (2003) afirma que a funcionalidade engloba todas as funçôes do corpo, o desempenho, as atividades e a participação social do indivíduo. 
Para Mello e Mancini (2007), a terapia ocupacional tem como objetivo de ação, frente ao paciente, a disfunção ocupacional, que é trazida no cotidiano do indivíduo como uma dificuldade para a realização de alguma atividade que lhe seja rotineira, independentemente se a causa para tal dificuldade é de ordem física, social, cognitiva ou outra. As autoras ainda completam que

[...] a habilidade ou a inabilidade de desempenhar as AVD (atividades de vida diária) podem ser usadas como medida prática para mensurar incapacidade (MELLO; MANCINI, 2007, p. 50).

Analisando-se as manifestações clínicas presentes na MPS II e percebendo que estas limitam a independência funcional destes indivíduos, fazendo com que sua qualidade de vida seja reduzida, foi analisado o nível de independência funcional destes portadores através da MIF, para que, assim, seja possível identificar quais as áreas de desempenho são afetadas mais rapidamente.

\section{Método}

Trata-se de um estudo transversal, descritivo, do qual participaram três adolescentes, irmáos, do sexo masculino, portadores de Mucopolissacaridose II, sendo um com 19 anos, outro com 16 anos e o último com 15 anos de idade, que se encontravam em tratamento genético pelo CER III (Centro Especializado em Reabilitação), localizado em Maceió-AL. Note-se que estes sujeitos eram os únicos portadores de MPS II registrados na instituição.

\subsection{Medida de Independência Funcional}

A aplicação do questionário foi realizada em uma sala reservada, numa instituição filantrópica, na qual os adolescentes realizam sessōes de fonoaudiologia, fisioterapia e terapia ocupacional, no município de Maceió-AL, sendo a entrevista conduzida por profissional devidamente treinado para utilização do instrumento. $\mathrm{Na}$ ocasião, permaneceram no local apenas o pesquisador principal, um auxiliar de pesquisa e a genitora do indivíduo pesquisado.

$\mathrm{O}$ instrumento de coleta de dados utilizado foi a Medida de Independência Funcional (MIF), que, de acordo com Riberto et al. (2012), o questionário tem por objetivo avaliar 18 níveis de funçóes e seus escores, os quais estão subdivididos em cuidados pessoais (alimentação, higiene matinal, banho, vestir - parte inferior, vestir - parte superior, uso do vaso sanitário); controles de esfíncteres (controle da urina, controle das fezes); mobilidade (transferência leito-cadeira, transferência vaso sanitário, transferência chuveiro); locomoção (locomoção horizontal, escadas); comunicação (compreensão, expressão), e cognição social (interação social, resolução de problemas, memória).

As respostas são objetivas e classificadas de 1 a 7, variando de acordo com o nível de auxílio que é preciso na execução daquela atividade, sendo: 7- independência completa; 6-independência modificada; 5 - supervisão; 4- ajuda mínima (realiza $>75 \%$ da atividade); 3 - ajuda moderada (realiza $>50 \%$ da atividade); 2 - ajuda máxima (realiza $>25 \%$ da atividade) e 1- ajuda total.

\subsection{Classificação socioeconômica}

Para definir a situação social dos sujeitos envolvidos na pesquisa, foi utilizado o critério de Classificação Econômica do Brasil, versão 2013 (ABEP, 2013), que categoriza os indivíduos, conforme a pontuação obtida, nas classes econômicas A1, A2, B1, B2, $\mathrm{C} 1, \mathrm{C} 2, \mathrm{D}$ e $\mathrm{E}$, que consiste em um somatório estabelecido a partir da posse de itens de consumo (televisão, rádio, banheiro, automóvel, geladeira e outros) e do grau de instrução do chefe da família, de maneira que, quanto maior o número de pontos obtidos, mais próxima à família estará da classe socioeconômica A e, em outra direção, a menor pontuação corresponde à classe $\mathrm{E}$. Esta classificação foi utilizada para, entre outros fatores, buscar relacionar a situação socioeconômica e o diagnóstico tardio, tendo em vista o acesso aos serviços de saúde e às informaçóes gerais sobre a doença.

A coleta de dados contou com a pesquisadora qualificada para aplicação do questionário. Ainda como parte do procedimento de pesquisa, a respondente foi esclarecida sobre os objetivos do estudo e solicitada a manifestar sua concordância, com a assinatura do Termo de Consentimento Livre e Esclarecido (TCLE), conforme as Normas Bioéticas de Pesquisa em Seres Humanos do Conselho do Ministério da Saúde do Brasil. Este estudo foi avaliado e aprovado por um Comitê de Ética em Pesquisa da Universidade Estadual de Ciências da Saúde de Alagoas - UNCISAL, com o protocolo de registro CEP n. ${ }^{\circ}$ 33297014.3.0000.5011.

\section{Resultados}

Para este estudo, foram avaliados três indivíduos, irmãos, portadores de Mucopolissacaridose II, do sexo masculino, negros e identificados por códigos: A1 - 19 anos; A2 - 16 anos e A3 - 15 anos. 
Os mesmos residem no município alagoano de Teotônio Vilela, que fica a aproximadamente $101 \mathrm{~km}$ da capital Maceió, local onde realizam as terapias (terapia enzimática, acompanhamento com geneticista e outras especialidades médicas, terapia ocupacional, fisioterapia e fonoaudiologia), uma vez por semana.

Todos foram encaminhados ao geneticista somente quando A1 já estava com sete anos de idade, devido à sintomatologia semelhante dos irmãos; na ocasião, A2 estava com quatro anos e A3 com três anos de idade.

De acordo com a classificação econômica proposta pela Associação Brasileira de Empresas de Pesquisa (2013), a família dos adolescentes estudados está inserida na classe $\mathrm{C} 1$, que compreende uma renda média de $\mathrm{R} \$ 1.685,00$, conforme o instrumento utilizado.

Estes recebem benefício social do governo, o Benefício de Prestação Continuada da Lei Orgânica da Assistência Social (BPC/LOAS), concedido pelo Instituto Nacional de Seguridade Social - INSS àqueles que apresentam alguma deficiência incapacitante ou possuem mais de 65 anos, e que comprovem não possuir meios de prover sua própria manutenção e de sua família. Este benefício tem cooperação direta com a manutenção familiar dos mesmos.

A Tabela 1 descreve as áreas de desempenho avaliadas através da MIF, obtidas por meio de entrevista com a mãe dos adolescentes, pela qual, através dos resultados, observou-se a condição progressiva da doença perante a dependência nas atividades de vida diária (AVD) e atividades instrumentais de vida diária (AIVD).

Considerando-se por ordem de nascimento, o filho mais velho, A1 (19 anos), já apresenta dependência total nas habilidades investigadas, passando a maior parte do dia em seu leito. $\mathrm{O}$ adolescente identificado como A2 (16 anos) apresenta perdas funcionais mais incapacitantes, necessitando de auxílio moderado, e o adolescente A3 (15 anos) ainda possui boa parte das habilidades físicas e cognitivas preservadas, sendo o único que ainda se mantém inserido na escola.

\section{Discussão}

A presente pesquisa teve como objetivo avaliar o nível de independência funcional dos portadores de Mucopolissacaridose II através de um estudo de corte transversal, com finalidade de analisar o desempenho ocupacional dos sujeitos envolvidos no estudo e contribuir para o entendimento das necessidades terapêuticas mais específicas dos indivíduos portadores de MPS II.

Quanto ao resultado obtido sobre a situação socioeconômica da família, pelas informaçóes sobre o local onde residem, associadas à escolaridade precária, observa-se que tal realidade afeta diretamente o acesso às informaçóes sobre a doença, o que pode ter tido como consequência o atraso no diagnóstico. Deve-se levar em consideração que, segundo a mãe dos sujeitos, a procura de um serviço de saúde para os irmáos mais novos foi realizada apenas pela semelhança com a sintomatologia do irmão mais velho. Diante desta discussão, levamos em conta a afirmação de Coelho e Rezende (2007), que relatam que, quanto mais precoce for detectado o atraso no desenvolvimento, mais rápida será a intervenção e, consecutivamente, menor o impacto de problemas na vida desta criança.

Segundo informaçôes fornecidas pela genitora, os três adolescentes são o quarto, o quinto e o sexto filhos de uma prole de seis, sendo os três primeiros do sexo feminino. A mesma relata que todos nasceram de parto normal e a termo, com peso adequado para a idade gestacional e, até os dois anos de idade, tiveram o desenvolvimento neuropsicomotor típico. Daí, então, começaram a apresentar dificuldades em realizar flexão dos dedos das mãos, com déficit na coordenação motora, rigidez articular, baixa estatura e abdome distendido. Relatou também que, à medida

Tabela 1. Caracterização da capacidade funcional dos adolescentes avaliados pela MIF.

\begin{tabular}{lccc}
\hline \multicolumn{1}{c}{$\begin{array}{c}\text { Categoria } \\
\text { (pontuação de referência) }\end{array}$} & A1* & A2** & A3*** \\
\cline { 2 - 4 } & & Pontuação obtida & 21 \\
Autocuidado (6-42) & 6 & 16 & 14 \\
Controle dos esfíncteres (2-14) & 2 & 8 & 21 \\
Transferências (3-21) & 3 & 18 & 8 \\
Locomoção (2-14) & 2 & 6 & 14 \\
Comunicação social (2-14) & 2 & 14 & 11 \\
Cognição social (3-21) & 4 & 5 & 89 (dependência \\
MIF total & 19 (dependência & 67 (dependência & modificada) \\
\hline
\end{tabular}

A1* - adolescente 1, 19 anos; A2** - adolescente 2, 16 anos; A3*** - adolescente 3, 15 anos. 
que os sintomas da doença apareciam, havia uma perda de habilidades anteriormente adquiridas.

Concordando com esses achados, os relatos de caso sobre a MPS II mostram que o curso da doença pode se dar através de desenvolvimento típico e, após certa idade, há uma regressão nas atividades que o indivíduo já desenvolvia com independência, ou apresenta atraso desde o início de seu desenvolvimento (PEREIRA et al., 2011; ROCHA et al., 2012).

Vieira et al. (2008) analisaram, em sua pesquisa, realizada entre maio de 2005 e novembro de 2006, no Serviço de Genética Médica do Hospital das Clínicas de Porto Alegre e em mais outros seis Serviços de Genética Médica no Brasil (sendo cinco no sudeste e um no nordeste), todo o histórico desde o nascimento até o diagnóstico bioquímico das Mucopolissacaridoses. Destas, a MPS II é o grupo que apresentou o maior atraso no desenvolvimento antes do diagnóstico e cuja sintomatologia apareceu mais precocemente. A MPS II foi ainda considerada como a MPS que apresentou maior atraso no diagnóstico, pois a idade média para o aparecimento dos sintomas é de 24 meses, enquanto a média para o diagnóstico foi de 95 meses. Este estudo ainda traz que a maioria dos casos de Mucopolissacaridoses, analisados por eles, teve os encaminhamentos feitos a médicos geneticistas e estes profissionais fizeram o diagnóstico da doença, por meio de exames específicos.

Foi observado, nesta análise, que os irmãos apresentam necessidade progressiva de auxílio na realização de suas atividades de vida diária e atividades instrumentais de vida diária, tendo o mais velho a dependência de ajuda total para realizar suas AVD. Estes resultados fortalecem o que foi citado por Guarany (2011), que afirma que a Mucopolissacaridose é uma doença crônica e progressiva, fazendo com que seus portadores fiquem cada vez mais dependentes na realização de suas atividades de vida diária, apesar de seus sintomas poderem ser retardados quando se faz terapia enzimática e tratamento multiprofissional na área da reabilitação.

O sujeito identificado como A1 é o que possui necessidade de maior auxílio para a realização de suas atividades, obtendo pontuação mínima em todos os escores, exceto no item memória, na cognição social, nos quais pontuou com 2 , o que aponta necessitar de ajuda máxima, enquanto A2, 16 anos, totaliza 67 pontos, apresentando maior pontuação na comunicação social e a menor em cognição social, e A3, 15 anos, dispóe de 89 pontos, expressando maior necessidade de auxílio nas açóes de autocuidado. Os dois irmãos mais novos se enquadram nos subescores de dependência modificada, o qual carece de assistência de até $25 \%$ de auxílio da tarefa.
O escore em que A3 necessita de maior auxílio no cumprimento de atividades é o autocuidado, sendo este o sujeito mais jovem, o que levanta a hipótese de que é esta a área de desempenho ocupacional que apresenta perda funcional inicial. Em respaldo, Camargos et al. (2012) relatam que, em seu estudo, foi identificada a relação entre domínio de função física e o autocuidado e a mobilidade. A pesquisa realizada por Morini (2007) traz o levantamento de que, em portadores de Mucopolissacaridose II, o déficit é maior em membros superiores quando comparados aos inferiores; a partir disto, se faz a associação com a dificuldade que apresentam de cuidar de si próprios, já que, para tal, há uma necessidade maior de utilização destes membros. Ainda complementando com ideias de Morini (2007), esta afirma que o tratamento deve ser focado em membros superiores, já que representam a queixa principal dos sujeitos de sua pesquisa.

Dentre os fatores identificados, neste estudo, que prejudicam a execução das atividades de vida diária, está incluso o comprometimento motor e a redução de força muscular. Corroborando esta ideia, Guarany (2011) relata que outro fator que acomete a independência destes pacientes são as manifestaçóes clínicas da MPS II e a pouca amplitude de movimento, além da rigidez e das contraturas articulares, também causadas pelo acúmulo de GAG nestas regiōes, prejudicando a mobilidade, a locomoção e a realização de tarefas. Também, em conformidade, Morini (2007) retrata um estudo realizado no ambulatório de Erros Inatos do Metabolismo do Hospital de Clínicas da Unicamp, no período entre fevereiro de 2005 e maio de 2006, com oito indivíduos portadores de Mucopolissacaridose II, sexo masculino, entre 4 e 46 anos, em que todos apresentaram perda de força em ao menos um grupo muscular, afetando-os na execução de suas atividades de vida diária. Sabe-se que a experiência motora está diretamente relacionada à aprendizagem cognitiva, sendo as descobertas familiarizadas através de tentativas, ou seja, se o componente motor está comprometido, consequentemente haverá alteraçốes cognitivas.

O comprometimento intelectual também foi identificado como outro fator que interfere na independência destes adolescentes, por estar diretamente relacionado com a compreensão daquilo que deve ser efetuado.

A Rede MPS Brasil (2011) afirma que a Mucopolissacaridose tipo II apresenta acúmulo de GAG no cérebro, o que traz como consequência a perda de habilidades adquiridas e o atraso no desenvolvimento; há, ainda, outros problemas, como a hidrocefalia, que podem dificultar o aprendizado, 
além de níveis inadequados de oxigênio e privação de sono (causado pela apneia do sono), que podem afetar a função cerebral.

De acordo com Guarany (2011), a identificaçáo da redução da independência de suas atividades cotidianas e das restriçóes na participaçáo social possibilita que os pacientes com MPS possam ser estimulados e recebam o acompanhamento adequado às suas necessidades.

Os sujeitos desta pesquisa possuíam ou possuem papéis ocupacionais comuns a crianças e adolescentes, porém, com o avanço da sintomatologia da MPS, foram se afastando e até mesmo perdendo estes papéis que faziam parte de suas vidas. Barrett e Kielhofner (2002) relatam que comportamento ocupacional envolve as atividades corriqueiras desenvolvidas pelo ser humano e que, quando esta ocupaçáo náo é permitida por algum fator, o sentimento de competência pode ficar ameaçado. Completando este pensamento, Beer (2002) afirma que o desempenho ocupacional pode ser afetado por patologias ativas, o que evidencia o quadro observado neste estudo.

A terapia ocupacional auxilia as pessoas na realização de atividades que são importantes para elas, apesar de suas debilidades e incapacidades, ampliando sua autonomia e reinserindo-a na comunidade/família (SOARES, 2007; NEISTADT; CREPEAU, 2002). Para que o terapeuta ocupacional possa reabilitar, através de atividades cotidianas, é necessário que ele avalie o contexto em que o paciente está inserido. Suas crenças e convicçóes, o meio em que vive e a dinâmica familiar são apenas alguns dos itens analisados por terapeutas ocupacionais para o planejamento do plano de tratamento mais adequado para cada indivíduo. É importante também não superestimar ou subestimar as capacidades do indivíduo e, se ele próprio o fizer, desenvolver um trabalho para que adquira confiança é fundamental em seu tratamento.

De acordo com Rogers e Holm (2002), o princípio mais utilizado para analisar o nível de incapacidade de alguém é o grau de independência apresentado na realização de uma tarefa. Arrematando esta consideração, Holm, Rogers e James (2002) trazem que o tratamento proposto pela terapia ocupacional aos sujeitos é a aquisiçáo de maior independência na execução de suas atividades de vida diária. Pedretti e Early (2005) citam que, pelo fato de a obtenção de independência ser o eixo central do processo terapêutico ocupacional, o método de intervenção deve seguir pelas conquistas nas áreas de desempenho do paciente, visando ao retorno aos seus papéis sociais.

Até o momento atual, não há cura para as Mucopolissacaridoses e, para o tratamento, devem ser considerados inicialmente a identificaçáo, a compreensáo e o manejo, analisando caso a caso quais as necessidades que mais se destacam em cada paciente e aliar essa conduta terapêutica ocupacional ao tratamento enzimático, buscando, essencialmente, o aumento da qualidade de vida através do retardo da progressão da doença (CAMELIER, 2011; REDE..., 2011; GIUGLIANI et al., 2010; GUARANY, 2011). Diante desta afirmação, é possível dizer que os métodos de tratamento adotados pelos pacientes pesquisados seguem a linha adotada por outros pesquisadores.

\section{Conclusão}

Apesar de poucos estudos relatarem as dificuldades motoras e cognitivas de portadores de Mucopolissacaridose II, foi observado, nesta pesquisa, que as áreas de desempenho ocupacional destes indivíduos sofrem perdas progressivas, corroborando com os achados da literatura. Tais perdas levam esses portadores à dependência funcional ainda na adolescência, considerando que, diante dos resultados obtidos nesta pesquisa, o autocuidado, a locomoção e a cognição social são as primeiras áreas do desempenho a apresentar perdas, visto que, segundo a avaliação do indivíduo mais jovem, essas áreas já estáo muito comprometidas, e nos seus irmãos mais velhos, já há perda maior da capacidade de realizar tarefas de modo independente.

Assim, ressalta-se a importância do diagnóstico precoce, visando estabelecer uma intervenção terapêutica imediata, com o objetivo de retardar as perdas funcionais, possibilitando que os indivíduos acometidos pela Mucopolissacaridose II possam manter-se o máximo possível de tempo realizando as suas atividades de vida diária com o mínimo de auxílio possível, potencializando, dessa forma, a qualidade de vida.

\section{Referências}

ASSOCIAÇÃO BRASILEIRA DE EMPRESAS DE PESQUISA - ABEP. Critério de classificação econômica Brasil. Sáo Paulo, 2013. Disponível em: <http://www. abep.org>. Acesso em: 17 maio 2013.

BARRETT, L.; KIELHOFNER, G. Teorias derivadas de perspectivas do comportamento ocupacional: uma visão geral do comportamento ocupacional. In: NEISTADT, M.; CREPEAU, E. B. Terapia Ocupacional: Willard \& Spackman. Rio de Janeiro: Guanabara Koogan, 2002. p. 488-489.

BEER, D. W. A Experiência da doença e da invalidez sob uma perspectiva individual. In: NEISTADT, M.; CREPEAU, E. B. Terapia Ocupacional: Willard \& Spackman. Rio de Janeiro: Guanabara Koogan, 2002. p. 27-36. 
BOY, R.; SCHWART, I. V. D. As doenças lisossômicas e o tratamento das Mucopolissacaridoses. Revista Hospital Universitário Pedro Ernesto, Rio de Janeiro, v. 10, p. 61-72, 2011. Suplemento 2.

CAMARGOS, A. C. R. et al. Relação entre a independência funcional e a qualidade de vida na paralisia cerebral. Revista Fisioterapia em Movimento, Curitiba, v. 25, n. 1, p. 83-92, 2012.

CAMELIER, M. V. Diagnóstico de Mucopolissacaridose tipo IVA em amostras de sangue impregnado em papel filtro. 2011. 96 f. Tese (Mestrado em Ciências Médicas) - Universidade Federal do Rio Grande do Sul, Rio Grande do Sul, 2011.

COELHO, Z. A. C.; REZENDE, M. B. Atraso no desenvolvimento. In: CAVALCANTI, A.; GALVĀO, C. Terapia Ocupacional: fundamentação \& prática. Rio de Janeiro: Guanabara Koogan, 2007. p. 299-305.

GIUGLIANI, R. et al. Terapia de reposição enzimática para as Mucopolissacaridoses I, II e VI: recomendaçóes de um grupo de especialistas brasileiros. Revista Associação Medica Brasileira, São Paulo, v. 56, n. 3, p. 257-277, 2010.

GUARANY, N. R. Avaliação do efeito da terapia de reposição enzimática na capacidade funcional de pacientes com Mucopolissacaridose. 2011. 120 f. Dissertação (Mestrado em Saúde da Criança e do adolescente) - Universidade Federal do Rio Grande do Sul, Porto Alegre, 2011.

HOLM, M. B.; ROGERS, J. C.; JAMES, A. B. Tratamento das áreas de desempenho ocupacional: tratamento das atividades de vida diária. In: NEISTADT, M.; CREPEAU, E. B. Terapia Ocupacional: Willard \& Spackman. Rio de Janeiro: Guanabara Koogan, 2002. p. 294-337.

JONES, K. L. Stronge disease. In: JONES, K. L. (Ed.). Padröes reconheciveis de malformações congênitas. São Paulo: Manole, 1998. p. 462-3.

MELLO, M. A. F.; MANCINI, M. C. Métodos e técnicas de avaliaçáo nas áreas de desempenho ocupacional. In: CAVALCANTE, A.; GALVÃO, C. Terapia Ocupacional: fundamentação \& prática. Rio de Janeiro: Guanabara Koogan, 2007. p. 49-52.

MORINI, S. R. Caracterização do sistema músculo-esquelético em individuos com Mucopolissacaridose tipo II: alguns aspectos cinéticos e consequências funcionais. $2007.82 \mathrm{f}$. Tese (Doutorado em Saúde da Criança e do Adolescente) - Universidade Estadual de Campinas, Campinas, 2007.

NERI, A. L. Palavras-chave em Gerontologia. Campinas: Editora Alínea, 2001.

NEISTADT, M. E.; CREPEAU, E. B. Introdução a Terapia Ocupacional. In: NEISTADT, M. E.; CREPEAU,
E. B. Terapia Ocupacional: Willard \& Spackman. Rio de Janeiro: Roca, 2002. p. 3-9.

NEUFELD, E. F.; MUENZER, J. The mucopolyssacaridoses. In: SCRIVER, C. R. et al. (Ed.). The metabolic and molecular basis of inherited disease. New York: McGraw-Hill, 2001. p. 3421-3452.

ORGANIZAÇÃO MUNDIAL DA SAÚDE - OMS. CIF: Classificação Internacional de Funcionalidade, Incapacidade e Saúde. São Paulo: USP, 2003.

PEDRETTI, L. W.; EARLY, M. B. Desempenho Ocupacional e modelos de prática para disfunção física. In: PEDRETTI, L. W.; EARLY, M. B. Terapia Ocupacional: capacidades práticas para as disfunçôes físicas. São Paulo: Roca, 2005. p. 3-13.

PEREIRA, E. C.; SACOMANI, D. G.; MOTTA, A. A. P. Manifestaçóes clínicas na Mucopolissacaridose do tipo II grave: relato de caso. Revista de Neurociências, São Paulo, v. 19, n. 4, p. 675-680, 2011.

PINTO, I. L. C. Uma estudo sobre a história natural da mucopolissacaridose tipo II (Sindrome de Hunter) em pacientes brasileiros. 2005. 125 f. Tese (Mestrado em Genética e Biologia Molecular) - Universidade Federal do Rio Grande do Sul, Porto Alegre, 2005.

PINTO, L. L. et al. Prospective study of 11 Brazilian patients with mucopolysaccharidosis II. Jornal Pediatria, Rio de Janeiro, v. 82, n. 4, p. 273- 278, 2006.

REDE MPS BRASIL. 2011. Disponível em: <http:// www.ufrgs.br/redempsbrasil/>. Acesso em: 11 out. 2014.

RIBERTO, M. et al. Validação da versão Brasileira da Medida de Independência Funcional. Acta Fisiátrica, São Paulo, v.11, n. 2, p. 72-76, 2012.

ROCHA, J. S. M. et al. Avaliação do desempenho motor em crianças com Mucopolissacaridose II. Cadernos de Terapia Ocupacional da UFSCar, São Carlos, v. 20, n. 3, p. 403-412, 2012.

ROGERS, J.; HOLM, M. B. Avaliação das áreas de desempenho Ocupacional. In: NEISTADT, M.; CREPEAU, E. B. Terapia Ocupacional: Willard \& Spackman. Rio de Janeiro: Guanabara Koogan, 2002. p. 167-201.

SOARES, L. B. T. História da Terapia Ocupacional. In: CAVALCANTI, A.; GALVÃO, C. Terapia Ocupacional: fundamentaçáo \& prática. Rio de Janeiro: Guanabara Koogan, 2007. p. 3-8.

VIEIRA, T. et al. Mucopolysaccharidoses in Brazil: what happens from birth to biochemical diagnosis? American Journal Med Genet A, Hoboken, v. 146A, n. 13, p. 1741$1747,2008$.

\section{Contribuição dos Autores}

Ingrid Alves Barros Silva Amaral foi responsável pela coleta de dados e redação do texto. Reinaldo Luna de Omena Filho foi responsável pela concepção e revisão do texto. João Ancelmo dos Reis Neto foi responsável pela traduçấo e organização das fontes. Monique Carla da Silva Reis foi responsável pela coleta de dados, análise e concepção do texto. Todos os autores aprovaram a versão final do texto. 